\title{
A scale for measuring the severity of diagnostic errors in accident and emergency departments
}

\author{
H R Guly
}

\begin{abstract}
Objective-To design and test a simple scale for measuring the severity of diagnostic errors occurring in accident and emergency (A\&E) departments.

Methods-Empirical design of a scale which indicates the severity of errors on a scale of 1 to 7 . It is obtained by adding two scores which indicate the additional treatment which a patient would have received and the follow up which would have been organised if the correct diagnosis had been made initially.

Results-The misdiagnosis severity score (MSS) revealed 166 diagnostic errors in injuries treated in an A\&E department over one year. The scoring system allowed the more significant errors to be separated from the less significant ones. Conclusions-The MSS proved useful in describing the errors made in an $A \& E$ department.

(f Accid Emerg Med 1997;14:290-292)
\end{abstract}

Keywords: accident and emergency department; misdiagnosis; severity scoring; trauma

Establishing a correct diagnosis is fundamental to good medical care and in any specialty misdiagnosis may lead to incorrect treatment and preventable morbidity and mortality. There are numerous papers describing diagnostic errors which occur in accident and emergency (A\&E) departments. Many are case reports of specific injuries but there are also larger studies describing misdiagnoses occurring in a set time $^{1}$ or specific errors such as misreading $x$ rays, ${ }^{2}$ failure to take $x$ rays, ${ }^{3}$ missed tendon injuries, ${ }^{4}$ or diagnostic errors in the multiply injured. ${ }^{5}$ With the current emphasis being placed on audit, the detection and monitoring of diagnostic errors should be part of every $A \& E$ department's audit programme.

While many diagnostic errors may be of little significance, others are more serious and so when discussing a diagnostic error it is important to be able to indicate its seriousness or significance. The words "significant" and "nonsignificant" have often been used ${ }^{6-8}$ but this division is subjective and does not differentiate between degrees of significance. A method of describing the significance of a diagnostic error is required. Wardrope and Chennells ${ }^{2}$ designed a severity score for use in their study of misdiagnosis of $x$ rays. This was based on the management decision made by the senior doctor at the time the error was brought to their attention. It can be summarised as:
No action required

False positive diagnosis

Symptomatic treatment needed

Treatment needed by GP

Outpatient treatment or follow up

Inpatient treatment needed

Life threatening errors

The advantage of this scale is that it is based on what actually occurred rather than on an opinion on what should have been done, but it has problems. The treatment at the time the injury is diagnosed is not necessarily the same as would have been given at the time the patient attended (for example, a skull fracture detected at 48 hours may require no treatment but had it been diagnosed initially, the patient would have been admitted and probably investigated with computerised tomography). In addition, it appears to make no allowance for the difference between admission for observation and admission for treatment, nor for injuries discovered at outpatient review or while the patient is an inpatient.

Factors which should be considered when determining the severity of a diagnostic error include

(1) the effect on the patient, for example: - Any threat to life, the potential for short term pain or disability and the potential for long term disability.

- The likelihood that the correct diagnosis can be made within a reasonable time. (A patient who has been admitted or brought back to the next review clinic is more likely to have the correct diagnosis made and correct treatment given earlier than the patient with the same injury who has been discharged.)

(2) the effect on the department, for example the risk of complaint or legal action as a result of the misdiagnosis.

These factors are interrelated.

When patients suffer as a result of a diagnostic error, they do so not because of the error itself but because the error leads to a failure to treat the injury. Treatment can be active- such as plaster, surgery, or drugs - but observation of the patient as an inpatient or outpatient to ensure early detection of complications, to maintain adequate symptom control, to reassure the patient, and so on, must also be regarded as treatment. The basis of a severity score for a missed injury should be a measure of the treatment which the patient failed to get.

To be of use, any severity score should be capable of being applied soon after an error is discovered. It should therefore reflect the potential for further problems and cannot be a measure of the actual consequences of an 
Table 1 Additional treatment score

1 No specific treatment other than advice

2 Support bandage/sling/simple medication/physiotherapy

3 Plaster of Paris/splint/IV insertion (for fluid or

drugs)/procedure under local anaesthetic or digital nerve block

4 Surgery under general or regional anaesthetic or other invasive procedure including chest drain, skeletal traction

5 Urgent surgery which should have been done immediately, for example for extradural haematoma, abdominal trauma

Table 2 Patient disposal score

1 Discharged or referred to general practitioner

2 Referred to outpatient clinic (including A\&E clinic)

3 Admitted or referred to other hospital

injury as these may not be known for months or even years.

\section{Methods}

On an empirical basis, a score to describe the severity of diagnostic errors on a scale of 1 to 7 was designed. This is made up of two components. The "additional treatment score" is a scale indicating the complexity of additional treatment which would have been given had the injury been correctly diagnosed at the first visit. This is shown in table 1 . The second component is a "patient disposal score" which is based on a scale used for producing health care resource groupings in $\mathrm{A} \& \mathrm{E} .{ }^{9}$ This is shown in table 2 .

The "misdiagnosis severity score" (MSS) is defined as the additional treatment score plus the difference between the patient disposal score relating to what would be been done had the injury been correctly diagnosed and what was actually done. Examples are given below.

\section{Example 1}

A compound depressed skull fracture is missed on $x$ ray and the patient is discharged.

Additional treatment required (surgery) 4

Disposal required (admit)

Disposal done (discharged)

Difference in disposal scores

Misdiagnosis severity score

$$
4+2=6
$$

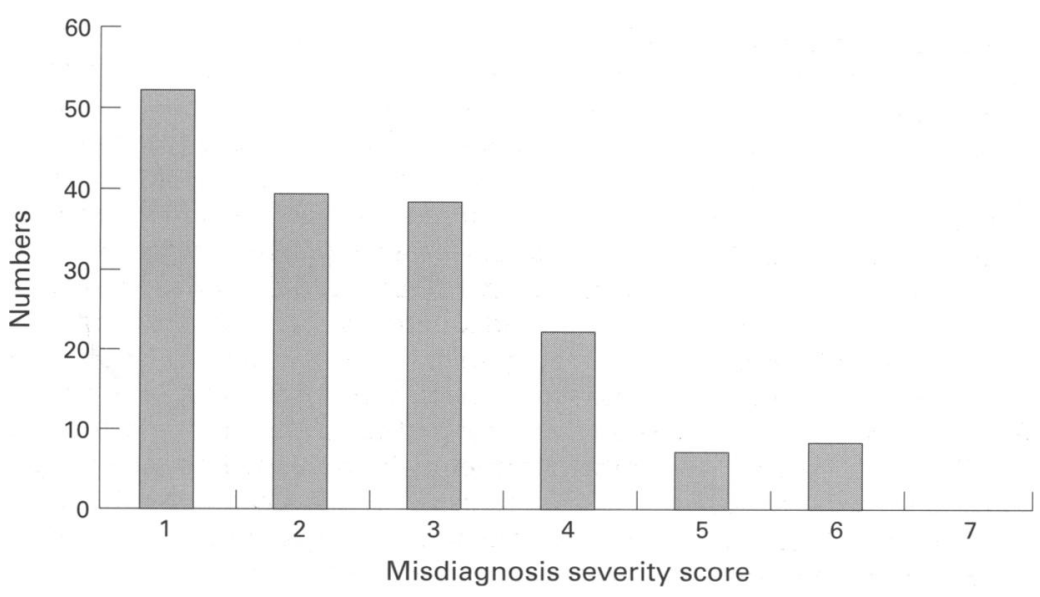

Figure 1 Misdiagnosis severity scores of the trauma diagnostic errors made in the accident and emergency department in 1995.
Example 2

An undisplaced fracture of the distal radius is missed but the patient had been treated in plaster and followed up as a clinical fracture of the scaphoid

Additional treatment required

(nil specific-already in plaster)

Disposal required (outpatient follow up) 1

Disposal done (outpatient follow up) 1

Difference in disposal scores

Misdiagnosis severity score

$1+0=1$

\section{Results}

In 1995 an audit in the A\&E department at Derriford Hospital revealed 176 diagnostic errors of which 166 were injuries and had an MSS applied. The severities are shown in fig 1 .

Scoring allows the more significant errors to be separated from the less significant. The diagnostic errors with a MSS of 5 and above are listed in table 3.

\section{Discussion}

The MSS is a scale which indicates the severity of diagnostic errors on a scale of 1 to 7 . No error scores zero as all errors have implications for patient care. This is because even if the medical consequences are minimal patients may be distressed that an error has been made and this can cause difficulty in their relations with the doctor or hospital. The MSS can be used on all errors within a given period as described above, or on subgroups such as misread $x$ rays, missed tendon injuries, or diagnostic errors in the multiply injured. I have only used the MSS to describe the severity of trauma diagnostic errors, but the same score could be used to describe the severity of non-traumatic diagnostic errors if additional treatment scores were allocated for nonsurgical treatments such as thrombolysis. The MSS is a non-linear scale. It must be emphasised that it is designed to describe the significance of diagnostic errors for the purposes of audit. It should not be used as an indicator of the consequences of an error for an individual patient, for example a missed flexor tendon injury will have greater consequences for a professional musician than for many other people.

The treatment of a particular injury may vary between hospitals. One unit may routinely treat a metatarsal fracture in plaster while another would usually treat the same injury in

Table 3 Misdiagnoses in 1995 with a misdiagnosis severity score (MSS) of 5 and above

\begin{tabular}{ll}
\hline MSS & Injuries \\
\hline 5 & $\begin{array}{l}\text { Rupture medial collateral ligament of knee }(\times 3) \\
\text { Pneumothorax } \\
\text { Digital nerve injury } \\
\text { Fracture calcaneum }\end{array}$ \\
Glass foreign body needing removal \\
Jefferson fracture C1 \\
Fracture odontoid \\
Compound fracture roof of orbit (penetrating injury) \\
Blow out fracture orbit \\
Bilateral fracture mandible \\
Rupture medial collateral ligament knee \\
Fracture neck of femur \\
Rupture ulna collateral ligament 1st \\
metacarpophalangeal joint
\end{tabular}


a supporting bandage. In most departments there are written guidelines on how to treat injuries but in practice these may be modified depending on factors such as the degree of pain or functional deficit, previous medical problems, and time since the injury. The treatment score of the missed injury should relate to the treatment recommended in the departmental guidelines unless a senior doctor believes that the treatment would have been modified. Interobserver variability has not been tested.

There are theoretical problems in adding two numbers both derived from non-linear scales, but the important factor is whether the MSS, however calculated, reflects the significance of diagnostic errors. The validation of this scale is described elsewhere. ${ }^{10}$

In summary, the MSS is simple, easy to use, and has proved useful in describing the severity of diagnostic errors in this department's audit programme.
1 Guly HR Missed diagnoses in an accident and emergency department. Injury 1984;15:403-6.

2 Wardrope J, Chennells PM. Should all casualty radiographs be reviewed? BMJ 1985;290:1638-40.

3 Guly HR. Fractures not Xrayed. Arch Emerg Med 1986;3: 159-62.

4 Guly HR. Missed tendon injuries. Arch Emerg Med 1991;8:87-91.

5 Chan RNW, Ainscow D, Sikorski JM. Diagnostic failures in the multiply injured. J Trauma 1980;20:684-7.

6 de Lacey G, Barker A, Harper J, Wignall B. An assessment of the clinical effects of reporting accident and emergency radiographs. Br J Radiol 1980;53:304-9.

7 Mucci B. The selective reporting of X-ray films from the accident and emergency department. Injury 1983;14: accident

8 Vincent CA, Driscoll PA, Audley RJ, Grant DS. Accuracy of detection of radiographic abnormalities by junior doctors. Arch Emerg Med 1988;5:101-9.

9 Brayley N, Marrow J. Health care resource groups in accident and emergency medicine. J Accid Emerg Med 1996;13:143-4.

10 Guly HR. The misdiagnosis severity score and doctors' perception of the severity of diagnostic errors. J Accid Emerg Med 1997;14:293-4.

Application forms are available from the Faculty of Accident and Emergency Medicine, The Royal College of Surgeons of England, 35 Lincoln's Inn Fields, London WC2; tel 01714057071 . Closing date for applications 31 October.

Applications are invited for the Alison Gourdie Memorial Scholarship. This award, to the value of $£ 1250$, is made annually to facilitate travel and/or research in the furtherance of emergency medicine. It is open to training grade doctors and consultants within five years of their appointment, and to any occupational group involved in the delivery of emergency care.

Application forms are available from the Faculty of Accident and Emergency Medicine, The Royal College of Surgeons of England, 35 Lincoln's Inn Fields, London WC2; tel 01714057071 . Closing date for applications 31 October. 